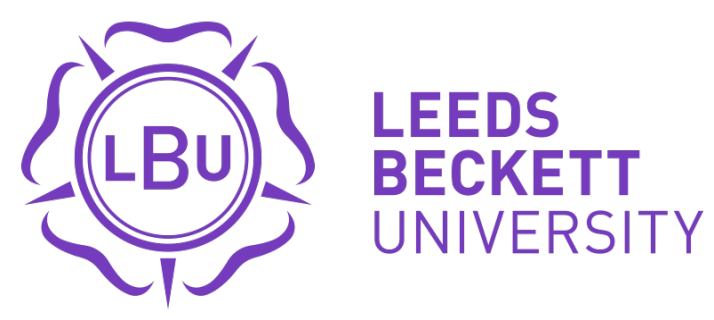

Citation:

Shah, $\mathrm{N}$ and Bye, $\mathrm{K}$ and Marshall, $\mathrm{A}$ and Woods, DR and O'Hara, JP and Barlow, M and Rimmer, J and Boos, C (2020) The Effects of Apnoea (hypoxia) Training, using voluntary Breath Holds, on High Altitude Adaptation: BREATHE-HA Study. High Altitude Medicine and Biology. ISSN 1527-0297 DOI: https://doi.org/10.1089/ham.2019.0087

Link to Leeds Beckett Repository record:

https://eprints.leedsbeckett.ac.uk/id/eprint/6541/

Document Version:

Article (Accepted Version)

Final publication is available from Mary Ann Liebert, Inc., publishers http://dx.doi.org/10.1089/ham.2019.0087

The aim of the Leeds Beckett Repository is to provide open access to our research, as required by funder policies and permitted by publishers and copyright law.

The Leeds Beckett repository holds a wide range of publications, each of which has been checked for copyright and the relevant embargo period has been applied by the Research Services team.

We operate on a standard take-down policy. If you are the author or publisher of an output and you would like it removed from the repository, please contact us and we will investigate on a case-by-case basis.

Each thesis in the repository has been cleared where necessary by the author for third party copyright. If you would like a thesis to be removed from the repository or believe there is an issue with copyright, please contact us on openaccess@leedsbeckett.ac.uk and we will investigate on a case-by-case basis. 


\section{The Effects of Apnoea Training, using Voluntary Breath Holds, on High Altitude Acclimation: BREATHE-HA Study}

Running title: Apnoea training and high altitude acclimation

Nishma Shah ${ }^{1}$, Kyo Bye ${ }^{2}$, Anna Marshall ${ }^{1}$, David R Woods ${ }^{2,3,4}$, John O’Hara ${ }^{3}$, Matthew Barlow ${ }^{3}$, R Rimmer², Christopher John Boos ${ }^{3,5,6}$

${ }^{1}$ Department of Surgical Sciences, Institute of Sports and Exercise Health, University College, London WC1E $6 B$

${ }^{2}$ Defence Medical Services, Lichfield, WS14 9PY

${ }^{3}$ Research Institute, for Sport, Physical Activity and Leisure, Leeds Beckett

University, Leeds, LS16 5LF

${ }^{4}$ Northumbria and Newcastle NHS Trusts, Wansbeck General and Royal Victoria Infirmary, Newcastle, NE66 2NS

${ }^{5}$ Department of Cardiology, Poole Hospital NHS Foundation trust, Poole, BH15 2JB

${ }^{6}$ Dept of Postgraduate Medical Education, Bournemouth University, Bournemouth, BH1 $3 L T$ 


\begin{abstract}
Introduction: There is evidence that intermittent hypoxic exposure (IHE) may improve high altitude (HA) performance. In this study the effects of short-term IHE via voluntary apnoea training on HA-related symptoms including acute mountain sickness (AMS) were examined for the first time.
\end{abstract}

Methods: Forty healthy adults were randomised to a self-administered apnoea training $(n=19)$ or to a control group ( $n=21$ no apnoea training) prior to ascent to an altitude of $5100 \mathrm{~m}$ in the Himalayas over 14 days. The apnoea training was conducted at sea level and consisted of five breath holds per day in week one, seven in week two, followed by 10 per day from weeks 3-6 and until HA exposure. Arterial oxygen saturation $\left(\mathrm{SpO}_{2}\right)$, heart rate, sleep quality (Insomnia Severity Index, ISI), rating of perceived exertion (RPE), blood pressure and Lake Louise Scores were measured at sea level (in the UK) and at HA at 1400m, 2700m, 3400-3700m, 4050-4200m, 4800m and 5100-5200m. Anxiety (GAD-7) scores were examined at sea level, $1400 \mathrm{~m}$ and at $5100-5200 \mathrm{~m}$

Results: Apnoea training led to a significant improvement in the mean longest breathhold times from baseline $(80.42 \pm 32.49$ [median 87.00 ] seconds) to the end of week six $(107.02 \pm 43.65[113.00]$ seconds) respectively $(\mathrm{p}=0.009)$. There was no significant difference in the prevalence of AMS $(8 / 19=42.1 \%$ vs $11 / 21=52.4 \%$; RR $0.80 ; 95 \%$ CI 0.41- 1.57: $\mathrm{p}=0.80$ ) or in GAD-7, ISI and RPE, $\mathrm{SpO}_{2}$, heart rate or blood pressure among the apnoea versus control groups respectively at HA. 
Conclusions: Apnoea training does not lessen HA-related symptoms in healthy adults travelling up to $5200 \mathrm{~m}$. Larger studies using more challenging apnoea protocols and to higher altitudes should be considered.

Key words: High altitude, acute mountain sickness, apnoea training, anxiety, sleep, perceived exertion 


\section{Introduction}

The recent improvements in infrastructure and access have led to significantly increasing numbers of persons travelling to high altitude (HA) than that of only a decade ago. HA-related symptoms including anxiety, insomnia and acute mountain

sickness (AMS) are some of the commonest obstacles to success. AMS can rapidly worsen and lead to the development of HA cerebral oedema (HACE) and even death if it is unrecognised and not treated (Bartsch and Swenson 2013; Boos et al., 2018). HA-related hypoxia can also cause HA pulmonary oedema (HAPE), which is a noncardiogenic form of pulmonary oedema resulting from excessive hypoxic pulmonary vasoconstriction, which can also be fatal if not recognised and treated promptly (Bartsch and Swenson 2013). Unfortunately, despite intense research into the field, there are no reliable sea level predictors to identify individuals who are at a particularly high risk of developing AMS and its complications among a general population of otherwise healthy adults (Bartsch and Swenson 2013; Lawrence and Reid 2016; Masuet-Aumatell et al., 2017).

Consequently, there has been an increasing focus not only on methods to try to identify individuals at increased risk of HAPE and AMS, but to pre-emptively mitigate their risk "prophylactically". There is emerging, yet inconsistent, evidence to suggest that intermittent hypoxic exposure (IHE) can improve exercise performance and induce a degree of pre-acclimatisation (acclimation) that is associated with a reduction in the risk of AMS development and its severity (Dehnert et al., 2014; Fulco et al., 2013; Lizamore and Hamlin 2017; Molano Franco et al., 2019; Muza 2007; Wille et al., 2012). These studies have used either altitude chambers or low $\mathrm{FiO}_{2}$ breathing (hypoxicators) to replicate the hypoxia of genuine terrestrial HA. Both methods are expensive and logistically challenging to perform, thereby limiting their widespread 
utility. The mechanisms to explain their benefit appear to be multiple and are still poorly understood. Those cited include increased erythropoiesis, angiogenesis and release of circulating stem cells, reducing oxidative stress and systemic inflammation, improved autonomic and mitochondrial function, chemosensitivity to hypoxia and aerobic performance (Casas et al., 2000; Gangwar et al., 2019; Povea et al., 2005; Viscor et al., 2018). Its benefits appear to be heavily influenced by the population studied, the mode, frequency and intensity of the hypoxic stimulus as well exercise components (Hamlin et al., 2018; Viscor et al., 2018).

Voluntary breath holding (apnoea training), is another method of reproducing shortlived IHE that has been used successfully for $>25$ years in free divers to enhance their performance (Bain et al., 2018). It has the obvious advantage over chamberbased IHE in that there is no financial cost and it can be easily conducted in an individual's own time. There is published data to suggest its positive effects on both aerobic and anaerobic exercise performance (Lemaitre et al., 2010) . However, its impact on HA-related symptoms and performance has not been explored. Whilst the hypoxic period is far shorter with apnoeic training compared with conventional IHE it has been shown to induce a variety of physiological changes that could be potentially utilised to the benefit of HA acclimation. These include increased splenic volumes and contraction, increased circulating haematocrit and haemoglobin concentrations to potentially improve arterial oxygen content (Schagatay et al., 2001; Schagatay et al., 2005). Other published mechanisms include increased chemoresponsiveness, cerebral blood flow and the bradycardic response to apnoea (Bain et al., 2018). Together these factors may act to improve hypoxic resilience and lessen myocardial oxygen consumption whilst maintaining cerebral oxygenation which may 
be of translational benefit for HA acclimation (Costalat et al., 2014; Schagatay et al., 2001). Additional potential benefits include improved mindfulness and ventilatory control that may improve mood, anxiety and sleep quality (Cho et al., 2016; Lemaitre et al., 2010). It has been demonstrated that apnoeic duration and the magnitude of associated bradycardia are linked to a lower risk AMS supporting the potential translational impact of apnoea training to reduce HA-related illness (Austin and Sleigh 1995; Holmstrom et al., 2019).

In this study we aimed to investigate for the first time the effects of apnoea training on HA related symptoms. We hypothesised that apnoea training offers a simple method of personal acclimation that leads to improved performance at HA and a reduction in the burden of AMS.

\section{Population and Design}

This was a prospective intention-to-treat observational study of 42 healthy adult male $(n=30)$ and female $(n=12)$ British military service volunteers who were due to take part in RAF 100 Ex Himalayan Venture 18, to the Himalayas in September 2018. They were all low altitude dwellers without previous breath hold training experience. The population consisted of two trekking teams (TT) in the Khumbu valley region of Nepal undertaking an identical ascent and one Alpine Team (AT). The AT followed a marginally different ascent profile but along a similar altitude and commencing in the Rolwaling Valley. The subjects were assessed at baseline (sea level [SL]), 1400m (day-1), 2700m (day-2 for TT and day-3 for AT), 34003700m (day-3 for TT and day-4 for AT), 4050-4200m (day-5), 4800-4900m (day-7 
for AT and day- 8 for TT). Rest days were scheduled where there was an altitude gain of $\geq 1000 \mathrm{~m}$, hence days- 3 and 6 for the TT and days- 6 and 8 for the AT. Thereafter, the sleep altitudes ranged from 4000- $4996 \mathrm{~m}$ on days 9-14 before a further ascent to $5100-5200 \mathrm{~m}$ in both groups on day 14 . None of the participants had been exposed to simulated or terrestrial HA in the preceding six weeks prior to inclusion.

The primary study outcome measure was the development of AMS. Secondary outcome measures of interest were the comparative differences in heart rate, blood pressure, anxiety-related symptoms, sleep quality, peripheral arterial oxygen saturations $\left(\mathrm{SpO}_{2}\right)$ and rating of perceived exertion (RPE).

All participants were engaged in regular physical activity and had passed their Service Specific fitness tests and were fully fit for military duty. A modified version of the Centre of Aviation Medicine (RAF Henlow) health questionnaire was used for the health screening of the participants prior to their inclusion.

\section{Randomisation and Apnoea Protocol}

The population of research volunteers were randomised to one to one of two research groups - a control group and an apnoea group.

The apnoea protocol was conducted at SL and consisted of five breath holds per day in week one, seven in week two, followed by 10 per day from weeks 3-6 and at least 
until their departure to HA. This protocol was designed following a previous study at HA (Mellor et al., 2017) and its demonstration of an induction in splenic contraction in a previous sea level apnoea study (Schagatay et al., 2005). The apnoea protocol consisted of two minutes of calm and controlled breathing at a rate of 5-6 breaths per minute, followed by a deep, but not maximal inspiration before each apnoea. The apnoeas were conducted in a static sitting or lying position. The protocol mandated that apnoea training be performed for five out of seven days of each week. Participants in the apnoeic group were supplied with a breath hold diary to record their longest breath hold time and fingertip pulse oximeter in order to monitor their oxygen desaturation, during apnoeas, and were instructed to interrupt their breath hold if their $\mathrm{SpO}_{2}$ levels fell to $\leq 60 \%$ as previously described (Engan et al., 2013). They were instructed to document their lowest $\mathrm{SpO}_{2}$ readings for each day of apnoea training whilst at SL. None of the participants in either group had received previous apnoea training prior to this study.

\section{Data collection and Measurements}

Baseline data was collected at effective SL (44m) 70 days prior to departure. Thereafter data collection was performed at 1400m, 2700m, 3400-3700m, 40504200m, 4800m and 5100-5200m.

HA-related symptoms were recorded twice daily using the updated 2018 Lake Louise Scoring (LLS) system (Roach et al., 2018). The total LLS score for an individual was calculated as the sum of the scores for the four symptoms (headache, nausea/vomiting, fatigue, and dizziness/light-headedness). AMS was defined as a 
total score of at least three points which included at least one score for headache at an altitude of $\geq 2000 \mathrm{~m}$ and a recent height gain (Roach et al., 2018). The subjects were advised not to take prophylactic acetazolamide. However, acetazolamide use was permitted, under medical guidance (by the expedition doctor) for the treatment of AMS. The use of non-steroidal anti-inflammatory medication and paracetamol was permitted and documented.

The Borg RPE was recorded at the end of each day (Borg 1970). This is a 15 point numerical scale numbered from 6-20, with values of 6 representing the resting state and 20, exhaustive exercise that has been used at HA previously (Borg 1970). The highest RPE during the day was recorded to reflect the overall effort. The other physiological variables were recorded twice daily, pre breakfast and before evening meal and at least 3 hours after arrival at each new altitude. Blood pressure was measured on fully rested and seated subjects using a brachial cuff fitted to the dominant arm using the USCOM BP ${ }^{+}$Device (Uscom, Sydney, NSW, Australia). Resting oxygen saturations $\left(\mathrm{SpO}_{2}\right)$ at $\mathrm{HA}$ were measured from the finger at using a Nonin Onyx Series II pulse oximeter (Plymouth, MN 55441, USA).

Sleep quality was examined daily using the Insomnia Severity Index (ISI) prebreakfast the night after arriving at each altitude (Bastien et al., 2001). This is a short form questionnaire that was designed to assess the severity of both night-time and daytime components of insomnia. The ISI is a 7-item self-report questionnaire assessing the nature, severity, and impact of insomnia. The dimensions evaluated were the severity of sleep onset, sleep maintenance, early morning awakening problems, sleep dissatisfaction, interference of sleep difficulties with daytime 
functioning, noticeability of sleep problems by others, and distress caused by the sleep difficulties. A 5-point Likert scale was used to rate each item (e.g., $0=$ no problem; 4 = very severe problem) to yield a total score ranging from 0 to 28 . The total score was interpreted as follows: absence of insomnia (0-7); sub-threshold insomnia (8-14); moderate insomnia (15-21); and severe insomnia (>21) as previously described (Bastien et al., 2001).

Anxiety levels were quantified using the Generalised Anxiety Disorder Assessment (GAD-7) questionnaire (Spitzer et al., 2006) at sea level baseline and at 1400m (day 1) and day 14 above $5000 \mathrm{~m}$. It is a seven-item instrument that is used to measure or assess the severity of generalised anxiety disorder (GAD). Each item asks the individual to rate the severity of his or her symptoms over the past two weeks. The GAD-7 score is calculated by assigning scores of $0,1,2$, and 3 , to the response categories of "not at all", "several days", "more than half the days" and "nearly every day" respectively, and then adding together the scores for the seven questions with the total score ranging from 0 to 21 . Total scores were used to determine the severity of anxiety symptoms which were graded as mild (score of 5-9), moderate (10-14) and severe ( $\geq 15)$ (Spitzer et al., 2006).

\section{Ethics}

The study was approved by the Ministry of Defence Research Ethics Committee and was conducted according to the standards of the Declaration of Helsinki. All participants were required to provide written and informed consent prior to inclusion. 
Our sample size was estimated from previously published work. Schagatay et al (2005) demonstrated a significant $(\mathrm{p}<0.001)$ and $18 \%$ reduction in splenic volume (due to splenic contraction) following three successive apneas among ten healthy adults. Gangwar et al (2019) demonstrated a significant reduction in AMS and higher $\mathrm{SpO}_{2}$ at $\mathrm{HA}$ among 40 healthy male adults randomized to IHE $(n=20 ; 12 \%$ $\mathrm{FIO}_{2}$ for $4 \mathrm{~h}$ per day for 4 consecutive days) versus non treatment (ie controls, $\mathrm{n}=20$ ). The participants were examined at SL and for seven consecutive days after being airlifted to $3520 \mathrm{~m}$. We estimated that a similar sample size of at least 40 participants, exposed to a longer period of self-administered IHE (six weeks) and to higher altitudes (hence more AMS symptoms) would provide sufficient power to detect notable differences in LLS and AMS prevalence among those randomised to IHE versus no IHE (controls).

\section{Statistical Analysis}

Data was analysed using GraphPad Prism version 6.07 (GraphPad Software, San Diego, CA, USA; www.graphpad.com) and SPSS version $22\left(\mathrm{IBM}^{\circledR}\right)$. Data inspection and the D'Agostino-Pearson normality test was undertaken to assess normality of all continuous data. Results are presented as mean \pm standard deviation (SD). Categorical variables were compared using the Fishers exact and chi squared tests with $95 \%$ confidence intervals (CI). Independent two group comparisons of continuous data were analysed using an unpaired t-test or Mann Whitney test for parametric and nonparametric data respectively. Comparisons of continuous data from $\geq 3$ groups of parametric and non-parametric data were performed using a one-way ANOVA or Kruskal-Wallis test respectively. Correlation analyses were performed using Pearson 
Correlation and Spearman's rank correlation with 95\% CIs depending on data normality. Only correlations with an $\mathrm{R}>0.20$ were reported. A split level factorial ANOVA) was undertaken in SPSS to assess the main effects of apnoea training (yes or no), change in altitude, and potential interaction on the dependent outcomes. The normality of distribution, the homogeneity of covariance matrices and the independence and the sphericity assumptions were verified. Mauchly's test was used to check for sphericity. If the sphericity assumption was violated, the GreenhouseGeisser correction was applied to adjust for the degrees of freedom of the interaction effect between different time points and different sample groups. If the value was less than 0.75 , the Huynh-Feldt correction was used for repeated measures analysis of variance. Binary logistic regression was undertaken to identify the baseline factors that were predictive of AMS at HA. A p-value of $<0.05$ was considered significant for all comparisons.

\section{Results}

From the initial inclusion of 42 participants, two people from the control group were excluded: one lost their data collection booklets on the mountain and the other participant failed to complete their data collection. Hence, the final analysis cohort consisted of 40 participants which consisted of 19 in the apnoea group and 21 in the control group. Among the apnoea group eight completed the entire protocol without any missed sessions. Eleven participants missed one or more apnoea days as follows: $\leq 3$ apnoea days $(n=3)$, complete cessation after the first three weeks $(n=4)$, missing up to a week in the middle $(n=2)$, three weeks at end $(n=2)$. 
The apnoea and control groups were very similar in terms of age, sex, ethnicity and other baseline demographics (Table 1). There was no difference in their baseline $\mathrm{SpO}_{2}$, heart rate, blood pressure, sleep and GAD-7 scores (Table 1). Apnoea training led to a significant improvement in longest breath-hold times from baseline $(80.42 \pm 32.49$ [median 87.00] seconds) to the end of week $6(107.02 \pm 43.65$ [median 113.00] seconds; $\mathrm{p}=0.009)$. Their longest breath-hold time $(134.94 \pm 56.04$ seconds) significantly increased compared with their baseline values $(\mathrm{p}=0.0002)$.

HA exposure led to a significant increase in resting heart rate, systolic and diastolic blood pressure, LLS (Figure 1), Borg and GAD-7 Scores and a reduction in $\mathrm{SpO}_{2}$ (Figure 2) (Table 3). Out of the 40 subjects 28 had no significant anxiety symptoms (scores of 0-4), symptoms were mild in 8 (score 5-9) and at least moderate in 4 (Scores of $\geq 10)$ at Day $14(5100 \mathrm{~m})$ There was no significant change in ISI scores.

At $\mathrm{HA}, \mathrm{SpO}_{2}$ inversely correlated with heart rate $(\mathrm{r}=-0.21 ; 95 \% \mathrm{CI}-0.30$ to -0.12 : $\mathrm{p}<0.0001)$ and RPE ( $r=-0.60 ;-0.68$ to $-0.52: \mathrm{p}<0.0001)$. GAD-7 scores correlated with RPE ( $\mathrm{r}=0.29 ; 95 \%$ CI 0.06 to $0.48: \mathrm{p}=0.01)$ and ISI scores $(\mathrm{r}=0.39 ; 0.18$ to 0.56 : $\mathrm{p}=0.0004)$

Overall $19(47.5 \%)$ participants experienced AMS at one or more altitudes $\geq 2000 \mathrm{~m}$. There was no significant difference in the primary study outcome of AMS prevalence among the apnoea group $(8 / 19=42.1 \%)$ versus controls $(11 / 21=52.4 \%$; RR $0.80 ; 95 \%$ CI 0.41 to 1.57: $\mathrm{p}=0.80$ ). Eight participants from the apnoea group and six from the control group were treated with Acetazolamide $(\mathrm{p}=0.75)$. None of the measured SL variables were predictive of AMS at HA. Four participants from the apnoea group and four in the control group were treated with non-steroidal medication and/or paracetamol $(\mathrm{p}=1.0)$. 
Apnoea training (versus controls) also did not influence the secondary outcomes of GAD-7 scores (or prevalence of anxiety, GAD $\geq 5$ ), ISI scores, RPE or other physiological measures including heart rate and $\mathrm{SpO}_{2}$. Furthermore, there was no evidence of an interaction of apnoea training-X-altitude on any of the main outcome variables. (Table 3)

The eight participants who completed the full apnoea training protocol were again similar in demographics to the control group. Among this apnoea group the mean $\left( \pm\right.$ SD [range]) daily minimal $\mathrm{SpO}_{2}$ readings during apnoeas were significantly lower over week six $(88.25 \pm 8.85 \%$ [61-98\%]) versus week one $(91.50 \pm 6.65 \%$ [74-99]; $\mathrm{p}=0.015$ ). Conversely, their longest breath-hold times also significantly increased from baseline $(82.77 \pm 34.96$ [median 88.40] seconds) to the end of week six (109.58 \pm 55.12 [107.40] seconds) respectively (overall ANOVA $\mathrm{p}=0.009)$. On the split-level repeated measures ANOVA again there was no significant differences between this group ( $\mathrm{n}=8$ ) versus the control group on any of the main study outcomes or in the development of AMS. 


\section{Discussion}

This is the first prospective randomised study to investigate the effects of apnoea training on the development of HA-related symptoms and AMS. In this study apnoea training was not associated with a reduction in AMS symptoms or severity. Apnoea training did not improve sleep quality, anxiety levels, RPE, resting heart rate, brachial blood pressure or $\mathrm{SpO} 2$.

The premise of this study was based on published evidence over the last 15 years supporting a role for IHE to augment exercise performance across a wide range of sporting disciplines (Czuba et al., 2017). There is also emerging data to suggest that IHE can induce acclimation effects, with the potential to reduce HA-related illness (Gangwar et al., 2019; Muza 2007). This is an extremely exciting prospect given the enormous clinical burden of AMS, which is known to affect $>40 \%$ of all persons travelling above 3500m (Bartsch and Swenson 2013; Boos et al., 2018). AMS remains the commonest medical condition encountered at HA and can rapidly progress to HACE and even death (Bartsch and Swenson 2013). The underlying concept behind IHE is its utility to induce hypoxic preconditioning and lessen the deleterious effects of hypoxia. Despite its promise, the published data is inconsistent. This may in part relate to the marked heterogeneity in the IHE protocols used in these studies. They have varied widely in terms of the hypoxic mode used (normobaric versus hypobaric hypoxia versus voluntary apnoea), its severity, duration (seconds to hours) and the number of cycles used (Gangwar et al., 2019; Hamlin et al., 2018; Muza 2007).

In our study we investigated whether apnoeic training, using voluntary breath holds, can deliver effective IHE and translate into better acclimatisation at HA and lessen 
HA-related symptoms (including AMS). Apnoeic training is proven to be effective in terms of improving free diving performance with some data to support its additional role in enhancing normoxic (ie sea level) athletic performance (Bain et al., 2018; Lemaitre et al., 2010). The cited mechanisms for its performance enhancing effects include reducing blood acidosis, oxidative stress, basal metabolic and heart rate, increasing erythropoietin concentration and reticulocyte counts, enhanced splenic volume and contraction, lung volumes and anaerobic glycolysis activity as well improving cerebral blood flow, autonomic control and heart rate variability (Christoforidi et al., 2012; Engan et al., 2013; Inoue et al., 2013; Joulia et al., 2002; Lemaitre et al., 2010).

Our desire to investigate the impact of apnoeic IHE on sleep and anxiety stems from our own previous work and that of others (Boos et al., 2018; Dong et al., 2013). We have recently observed that anxiety symptoms were independent predictors of AMS and its severity among a cohort of 80 healthy adults trekking to $5140 \mathrm{~m}$ (Dong et al., 2013). Anxiety-related symptoms are frequently exacerbated at HA and are strongly linked to both somatic symptoms and sleep quality (Dong et al., 2013). Indeed, we observed a significant increase in anxiety scores at HA versus SL in this study. There was significant, though modest, correlation between GAD-7 anxiety and ISI scores. Unfortunately, apnoeic training did not improve either of these scores. We had hypothesised that the controlled breathing which was integral to the apnoeic 'warm up' might have led to a degree of mindfulness and relaxation training that could have improved anxiety and sleep scores. It is worth noting that in this study the impact of HA on sleep quality was marginal and less than we might have anticipated which could reflect the gradual ascent profile used. 
There are many plausible reasons to explain the negative results of this study. Compliance with the apnoea protocol was poor and this, in itself, could have been sufficient to explain the lack of observed benefits. In our protocol apnoea training was conducted in five out of seven days in the week. Hence, the two rest days could have mitigated any acclimation effects. Consequently, we do not know whether a more intense apnoea protocol without rest days might have led to different results. Voluntary breath holding is uncomfortable and requires significant motivation. We did not include any encouragement methods to maintain compliance which is a limitation (van der Zwan et al., 2015). Another consideration is that the apnoea intensity was perhaps insufficient to achieve a decent hypoxic stimulus. In a very recently published study, Bouten et al observed that whilst eight weeks of apnoeic training (five apnoeas per day and similar to our protocol) led to an increase in splenic volume it was not sufficient to elicit significant training acclimation (Bouten et al., 2019). The mean daily lowest $\mathrm{SpO}_{2}$ recordings during week six of apnoeic training at SL in our study was certainly not insignificant at $88.25 \%$. In fact, this is very similar to the average resting $\mathrm{SpO}_{2}$ recorded at $3400-3700 \mathrm{~m}$ but may still be an insufficient stimulus

Another factor that needs consideration regarding the modest hypoxia with breath hold training in this study is the limitations of peripheral pulse oximetry itself. We used finger rather than central (e.g. earlobe) pulse oximetry to record $\mathrm{SpO}_{2}$ during breath-holds because of its ease of use, widespread use and to maintain consistency with $\mathrm{SpO}_{2}$ readings at $\mathrm{HA}$. However, there is a well-recognised time delay between the quantification of central hypoxia and its manifestation on peripheral pulse oximetry $\mathrm{SpO}_{2}$ readings (Lindholm et al., 2007; Pertzov et al., 2019). This can lead 
to significant differences in simultaneously measured central oximetry readings which are reported to be $\geq 6 \%$ lower with ear lobe versus finger readings (Lindholm et al., 2007). These differences may be even greater when the onset of hypoxia is rapid such as with breath-holding as in this study. Hence, it is possible that we have under appreciated the severity of induced hypoxia with apnoea training in this study.

The hypoxic period in the majority of IHE studies, (using normobaric or hypobaric hypoxic training) have generally been well in excess of 30 minutes for each training session and far longer than the apnoeic period of $<5$ minutes obtained in our study (Gangwar et al., 2019; Lizamore and Hamlin 2017). Perhaps the potential hypoxic pre-conditioning effects of apnoea training are too short lived to induce a noticeable sustained pre-acclimatisation effect (Richardson et al., 2009). Hypoxia is common to apnoeic training, IHE and HA exposure. However, with apnoea there is compensatory hypercapnoeic hypoxia whereas with IHE and genuine HA hypocapnoeic hyperventilation ensues; this leads to a progressive rise in end tidal $\mathrm{O}_{2}$ and subsequent fall in end-tidal $\mathrm{CO}_{2}$ which is a crucial to $\mathrm{HA}$ acclimatization (Lizamore and Hamlin 2017; West J.B. 2012). Hence, it is plausible that apnoea training may not increase chemoreceptor and carotid body sensitivity noted with traditional IHE, undermining some its acclimation potential.

This study has a number of strengths that should be acknowledged. This was a prospective and randomised study conducted at both SL and HA. Our sample size was larger than the majority of previously published apnoeic studies (Bouten et al., 2019; Christoforidi et al., 2012; Engan et al., 2013; Richardson et al., 2009). Our study power was considerably strengthened by the fact the participants were investigated at seven different altitudes across a wide range of physiological and 
wellness outcomes (GAD-7 [anxiety], ISI [sleep quality], RPE [perceived exertion] and LLS [AMS symptoms]).

\section{Limitations}

The poor compliance rate with the apnoea protocol is a major limiting factor that needs to be re-emphasised, although the absence of benefit among those who completed the full apnoeic protocol strengthens the validity of our results. Whilst the overall altitudes and time of trekking were similar between the three groups studied, one of the groups (with a similar proportion randomised to apnoea and non-apnoea subjects) had a slightly different ascent profile and resided at higher altitude for a longer period. We did not examine comparative haemoglobin and erythropoietin levels in order to gauge the degree of successful hypoxic preconditioning with breath hold training. Whilst the longest breath hold duration significantly increased with apnoea training, the average breath hold duration was relatively short, again suggesting poor compliance with the protocol. Only the lowest $\mathrm{SpO}_{2}$ readings during apnoea were recorded. Finally, the ascent rate in this study was modest, particularly between $4800-5200 \mathrm{~m}$. This may have contributed to the relatively low incidence of AMS and limited the potential impact of breath-hold training on HA-related symptoms.

\section{Conclusion:}

In this study IHE using voluntary breath holds did not reduce the burden and severity AMS. Breath hold training also failed to provide any comparative advantages in terms 
of lessening the impact of HA exposure on sleep quality, anxiety scores, RPE during exercise, heart rate and blood pressure over traditional non-IHE preparation. A larger study with a more aggressive protocol using compliance encouragement methods should be considered. 


\section{References}

Austin D, Sleigh J. (1995). Prediction of acute mountain sickness. Bmj 311,989-90.

Bain AR, Drvis I, Dujic Z, MacLeod DB, Ainslie PN. (2018). Physiology of static breath holding in elite apneists. Exp Physiol 103,635-651.

Bartsch P, Swenson ER. (2013). Clinical practice: Acute high-altitude illnesses. N Engl J Med $368,2294-302$

Bastien CH, Vallieres A, Morin CM. (2001). Validation of the Insomnia Severity Index as an outcome measure for insomnia research. Sleep Med 2,297-307.

Boos CJ, Bass M, O'Hara JP, Vincent E, Mellor A, Sevier L, Abdul-Razakq H, Cooke M, Barlow M, Woods DR. (2018). The relationship between anxiety and acute mountain sickness. PLoS One 13,e0197147.

Borg G. (1970). Perceived exertion as an indicator of somatic stress. Scand J Rehabil Med 2,92-8.

Bouten J, Caen K, Stautemas J, Lefevere F, Derave W, Lootens L, Van Eenoo P, Bourgois JG, Boone J. (2019). Eight weeks of static apnea training increases spleen volume but not acute spleen contraction. Respir Physiol Neurobiol 266,144-149.

Casas M, Casas H, Pages T, Rama R, Ricart A, Ventura JL, Ibanez J, Rodriguez FA, Viscor G. (2000). Intermittent hypobaric hypoxia induces altitude acclimation and improves the lactate threshold. Aviat Space Environ Med 71,125-30.

Cho H, Ryu S, Noh J, Lee J. (2016). The Effectiveness of Daily Mindful Breathing Practices on Test Anxiety of Students. PLoS One 11,e0164822.

Christoforidi V, Koutlianos N, Deligiannis P, Kouidi E, Deligiannis A. (2012). Heart rate variability in free diving athletes. Clin Physiol Funct Imaging 32,162-6.

Costalat G, Pichon A, Coquart J, Bauer F, Lemaitre F. (2014). Cardio-ventilatory responses to poikilocapnic hypoxia and hypercapnia in trained breath-hold divers. Respir Physiol Neurobiol 192,48-54. 
Czuba M, Wilk R, Karpinski J, Chalimoniuk M, Zajac A, Langfort J. (2017). Intermittent hypoxic training improves anaerobic performance in competitive swimmers when implemented into a direct competition mesocycle. PLoS One 12,e0180380.

Dehnert C, Bohm A, Grigoriev I, Menold E, Bartsch P. (2014). Sleeping in moderate hypoxia at home for prevention of acute mountain sickness (AMS): a placebo-controlled, randomized double-blind study. Wilderness Environ Med 25,263-71.

Dong JQ, Zhang JH, Qin J, Li QN, Huang W, Gao XB, Yu J, Chen GZ, Tang XG, Huang L. (2013). Anxiety correlates with somatic symptoms and sleep status at high altitudes. Physiol Behav 112-113,23-31.

Engan H, Richardson M, Lodin-Sundstrom A, van Beekvelt M, Schagatay E. (2013). Effects of two weeks of daily apnea training on diving response, spleen contraction, and erythropoiesis in novel subjects. Scand J Med Sci Sports 23,340-8.

Fulco CS, Beidleman BA, Muza SR. (2013). Effectiveness of preacclimatization strategies for high-altitude exposure. Exerc Sport Sci Rev 41,55-63.

Gangwar A, Pooja, Sharma M, Singh K, Patyal A, Bhaumik G, Bhargava K, Sethy NK. (2019). Intermittent normobaric hypoxia facilitates high altitude acclimatization by curtailing hypoxia-induced inflammation and dyslipidemia. Pflugers Arch. Hamlin MJ, Lizamore CA, Hopkins WG. (2018). The Effect of Natural or Simulated Altitude Training on High-Intensity Intermittent Running Performance in Team-Sport Athletes: A Meta-Analysis. Sports Med 48,431-446.

Holmstrom P, Mulder E, Sundstrom AL, Limbu P, Schagatay E. (2019). The Magnitude of Diving Bradycardia During Apnea at Low-Altitude Reveals Tolerance to High Altitude Hypoxia. Front Physiol 10,1075.

Inoue Y, Nakajima A, Mizukami S, Hata H. (2013). Effect of Breath Holding on Spleen Volume Measured by Magnetic Resonance Imaging. PLoS One 8,e68670. 
Joulia F, Steinberg JG, Wolff F, Gavarry O, Jammes Y. (2002). Reduced oxidative stress and blood lactic acidosis in trained breath-hold human divers. Respir Physiol Neurobiol $133,121-30$.

Lawrence JS, Reid SA. (2016). Risk Determinants of Acute Mountain Sickness and Summit Success on a 6-Day Ascent of Mount Kilimanjaro (5895 m). Wilderness Environ Med $27,78-84$

Lemaitre F, Joulia F, Chollet D. (2010). Apnea: a new training method in sport? Med Hypotheses 74,413-5.

Lindholm P, Blogg SL, Gennser M. (2007). Pulse oximetry to detect hypoxemia during apnea: comparison of finger and ear probes. Aviat Space Environ Med 78,770-3. Lizamore CA, Hamlin MJ. (2017). The Use of Simulated Altitude Techniques for Beneficial Cardiovascular Health Outcomes in Nonathletic, Sedentary, and Clinical Populations: A Literature Review. High Alt Med Biol 18,305-321.

Masuet-Aumatell C, Sanchez-Mascunano A, Santangelo FA, Ramos SM, Ramon-Torrell JM. (2017). Relationship between Smoking and Acute Mountain Sickness: A MetaAnalysis of Observational Studies. Biomed Res Int 2017,1409656.

Mellor A, Bakker-Dyos J, Howard M, Boos C, Cooke M, Vincent E, Scott P, O'Hara J, Clarke SB, Barlow M and others. (2017). The British Services Dhaulagiri Medical Research Expedition 2016: a unique military and civilian research collaboration. J R Army Med Corps 163,371-375.

Molano Franco D, Nieto Estrada VH, Gonzalez Garay AG, Marti-Carvajal AJ, ArevaloRodriguez I. (2019). Interventions for preventing high altitude illness: Part 3. Miscellaneous and non-pharmacological interventions. Cochrane Database Syst Rev 4,Cd013315.

Muza SR. (2007). Military applications of hypoxic training for high-altitude operations. Med Sci Sports Exerc 39,1625-31. 
Pertzov B, Brachfeld E, Unterman A, Gershman E, Abdel-Rahman N, Rosengarten D, Kramer MR. (2019). Significant Delay in the Detection of Desaturation between Finger Transmittance and Earlobe Reflectance Oximetry Probes during Fiberoptic Bronchoscopy: Analysis of 104 Cases. Lung 197,67-72.

Povea C, Schmitt L, Brugniaux J, Nicolet G, Richalet JP, Fouillot JP. (2005). Effects of intermittent hypoxia on heart rate variability during rest and exercise. High Alt Med Biol 6,215-25.

Richardson MX, de Bruijn R, Schagatay E. (2009). Hypoxia augments apnea-induced increase in hemoglobin concentration and hematocrit. Eur J Appl Physiol 105,63-8.

Roach RC, Hackett PH, Oelz O, Bartsch P, Luks AM, MacInnis MJ, Baillie JK. (2018). The 2018 Lake Louise Acute Mountain Sickness Score. High Alt Med Biol 19,4-6.

Schagatay E, Andersson JP, Hallen M, Palsson B. (2001). Selected contribution: role of spleen emptying in prolonging apneas in humans. J Appl Physiol (1985) 90,1623-9; discussion 1606.

Schagatay E, Haughey H, Reimers J. (2005). Speed of spleen volume changes evoked by serial apneas. Eur J Appl Physiol 93,447-52.

Spitzer RL, Kroenke K, Williams JB, Lowe B. (2006). A brief measure for assessing generalized anxiety disorder: the GAD-7. Arch Intern Med 166,1092-7.

van der Zwan JE, de Vente W, Huizink AC, Bogels SM, de Bruin El. (2015). Physical activity, mindfulness meditation, or heart rate variability biofeedback for stress reduction: a randomized controlled trial. Appl Psychophysiol Biofeedback 40,257-68.

Viscor G, Torrella JR, Corral L, Ricart A, Javierre C, Pages T, Ventura JL. (2018). Physiological and Biological Responses to Short-Term Intermittent Hypobaric Hypoxia Exposure: From Sports and Mountain Medicine to New Biomedical Applications. Front Physiol 9,814 . 
West J.B. SRB, Luks A.M., Milledge J.S. . (2012). High altitude medicine and physiology. 5th ed. London: CRC Press

Wille M, Gatterer H, Mairer K, Philippe M, Schwarzenbacher H, Faulhaber M, Burtscher M. (2012). Short-term intermittent hypoxia reduces the severity of acute mountain sickness. Scand J Med Sci Sports 22,e79-85. 
Table 1 Baseline Demographics

\begin{tabular}{|l|l|l|l|}
\hline & $\begin{array}{l}\text { Control group } \\
\text { (No Apnoea } \\
\text { exposure) }\end{array}$ & $\begin{array}{l}\text { Apnoea } \\
\text { Group }\end{array}$ & P value \\
\hline Number & 21 & 19 & \\
\hline Age & $38.52 \pm 7.70$ & $37.42 \pm 7.22$ & 0.64 \\
\hline $\begin{array}{l}\text { Sex } \\
\text { - Men, \% }\end{array}$ & $17(80.90 \%)$ & $13(68.40)$ & 0.47 \\
\hline - Women, \% & $4(19.10 \%)$ & $6(32.60 \%)$ & 0.26 \\
\hline Height, cm & $177.10 \pm 7.54$ & $173.70 \pm 10.86$ & 0.58 \\
\hline Weight, kg & $77.10 \pm 10.04$ & $74.79 \pm 16.39$ & 1.0 \\
\hline Body mass index, kg/m² & $24.52 \pm 2.20$ & $24.51 \pm 3.42$ & 0.22 \\
\hline Smoking & 0 & 17 & 0.23 \\
\hline $\begin{array}{l}\text { - current } \\
\text { - Ex } \\
\text { - never }\end{array}$ & 6 & 2 & \\
\hline $\begin{array}{l}\text { Ethnicity } \\
\text { - White } \\
\text { - Other }\end{array}$ & 15 & 16 & \\
\hline P values refer to comparison of control versus apnoea group at inclusion. & \\
\hline
\end{tabular}


Table 2 Comparative changes in Physiological indices and questionnaires with increasing high altitude

\begin{tabular}{|c|c|c|c|}
\hline & Control & Apnoea & P value \\
\hline $\begin{array}{l}\text { Heart rate/ minute } \\
- \text { SL } \\
-1400 \mathrm{~m} \\
-2700 \mathrm{~m} \\
-3400-3700 \mathrm{~m} \\
-4050-4200 \mathrm{~m} \\
-4800 \mathrm{~m} \\
-51005200 \mathrm{~m}\end{array}$ & $\begin{array}{l}69.69 \pm 10.36 \\
76.63 \pm 8.02 \\
73.75 \pm 10.81 \\
77.13 \pm 13.23 \\
85.001 \pm 12.85 \\
77.38 \pm 15.19 \\
85.63 \pm 18.99\end{array}$ & $\begin{array}{l}68.0 \pm 9.60 \\
77.43 \pm 12.73 \\
77.23 \pm 8.24 \\
70.54 \pm 8.23 \\
81.31 \pm 12.72 \\
78.69 \pm 13.41 \\
89.08 \pm 16.26\end{array}$ & 0.39 \\
\hline $\begin{array}{l}\text { Systolic blood pressure, } \mathrm{mmHg} \\
-\mathrm{SL} \\
-1400 \mathrm{~m} \\
-2700 \mathrm{~m} \\
-3400-3700 \mathrm{~m} \\
-4050-4200 \mathrm{~m} \\
-4800 \mathrm{~m} \\
-5100-5200 \mathrm{~m}\end{array}$ & $\begin{array}{l}124.50 \pm 9.30 \\
121.79 \pm 15.02 \\
129.71 \pm 14.55 \\
127.43 \pm 13.32 \\
129.07 \pm 13.88 \\
134.57 \pm 14.91 \\
131.71 \pm 15.28\end{array}$ & $\begin{array}{l}133.75 \pm 11.13 \\
129.42 \pm 11.31 \\
129.33 \pm 7.94 \\
126.17 \pm 10.43 \\
132.42 \pm 11.09 \\
141.33 \pm 14.60 \\
141.08 \pm 16.84\end{array}$ & 0.12 \\
\hline $\begin{array}{l}\text { Diastolic blood pressure, } \mathrm{mmHg} \\
-\mathrm{SL} \\
-1400 \mathrm{~m} \\
-2700 \mathrm{~m} \\
-3400-3700 \mathrm{~m} \\
-4050-4200 \mathrm{~m} \\
-4800 \mathrm{~m} \\
-5100-5200 \mathrm{~m}\end{array}$ & $\begin{array}{l}77.21 \pm 13.39 \\
77.36 \pm 12.79 \\
80.57 \pm 12.84 \\
79.43 \pm 6.27 \\
79.36 \pm 5.72 \\
84.29 \pm 9.49 \\
86.07 \pm 9.07\end{array}$ & $\begin{array}{l}83.17 \pm 6.59 \\
79.38 \pm 3.69 \\
85.33 \pm 7.52 \\
81.50 \pm 8.08 \\
82.83 \pm 5.37 \\
85.33 \pm 8.40 \\
88.83 \pm 5.98\end{array}$ & 0.08 \\
\hline $\begin{array}{l}\mathrm{SpO}_{2}, \% \\
-\mathrm{SL} \\
-1400 \mathrm{~m} \\
-2700 \mathrm{~m} \\
-3400-3700 \mathrm{~m} \\
-4050-4200 \mathrm{~m} \\
-4800 \mathrm{~m} \\
-5100-5200 \mathrm{~m}\end{array}$ & $\begin{array}{l}98.06 \pm 1.03 \\
95.29 \pm 1.57 \\
93.24 \pm 2.05 \\
87.94 \pm 3.13 \\
87.76 \pm 4.80 \\
82.76 \pm 5.30 \\
83.18 \pm 3.45\end{array}$ & $\begin{array}{l}97.62 \pm 0.87 \\
95.85 \pm 1.77 \\
93.23 \pm 1.79 \\
90.69 \pm 2.17 \\
86.53 \pm 2.18 \\
84.62 \pm 3.50 \\
84.62 \pm 3.28\end{array}$ & 0.75 \\
\hline $\begin{array}{l}\text { Borg RPE scores } \\
- \text { SL } \\
-1400 \mathrm{~m} \\
-2700 \mathrm{~m} \\
-3400-3700 \mathrm{~m} \\
-4050-4200 \mathrm{~m} \\
-4800 \mathrm{~m} \\
-5100-5200 \mathrm{~m}\end{array}$ & $\begin{array}{l}6.57 \pm 1.12 \\
8.33 \pm 2.69 \\
10.62 \pm 1.60 \\
12.76 \pm 1.30 \\
12.67 \pm 1.52 \\
12.19 \pm 2.06 \\
13.00 \pm 1.70\end{array}$ & $\begin{array}{l}6.56 \pm 1.26 \\
9.25 \pm 2.21 \\
10.50 \pm 1.55 \\
12.37 \pm 1.26 \\
12.19 \pm 2.00 \\
12.81 \pm 1.05 \\
13.30 \pm 1.54\end{array}$ & 0.73 \\
\hline $\begin{array}{l}\text { Sleep scores } \\
- \text { SL } \\
-1400 \mathrm{~m} \\
-2700 \mathrm{~m} \\
-3400-3700 \mathrm{~m} \\
-4050-4200 \mathrm{~m} \\
-4800 \mathrm{~m} \\
-5100-5200 \mathrm{~m}\end{array}$ & $\begin{array}{l}3.19 \pm 3.39 \\
2.81 \pm 3.22 \\
3.86 \pm 3.53 \\
3.95 \pm 3.94 \\
4.00 \pm 3.61 \\
4.43 \pm 4.20 \\
4.43 \pm 5.21\end{array}$ & $\begin{array}{l}5.06 \pm 4.23 \\
4.19 \pm 2.51 \\
5.88 \pm 5.30 \\
5.00 \pm 3.76 \\
4.25 \pm 3.64 \\
4.50 \pm 4.07 \\
4.56 \pm 4.47\end{array}$ & 0.31 \\
\hline $\begin{array}{l}\text { GAD-7 Scores } \\
\text {-SL } \\
-1400 \mathrm{~m} \\
-5100-5200 \mathrm{~m}\end{array}$ & $\begin{array}{l}0.00 \pm 3.26 \\
4.10 \pm 4.66 \\
2.43 \pm 3.11\end{array}$ & $\begin{array}{l}1.37 \pm 2.39 \\
2.37 \pm 2.85 \\
2.226 \pm 2.79\end{array}$ & 0.35 \\
\hline $\begin{array}{l}\text { Lake Louise Scores } \\
\text {-SL } \\
-1400 \mathrm{~m} \\
-2700 \mathrm{~m} \\
-3400-3700 \mathrm{~m}\end{array}$ & $\begin{array}{l}- \\
0.81 \pm 1.39 \\
0.90 \pm 1.18 \\
1.71 \pm 1.23\end{array}$ & $\begin{array}{l}- \\
1.37 \pm 1.74 \\
1.11 \pm 1.49 \\
1.68 \pm 1.11\end{array}$ & 0.47 \\
\hline
\end{tabular}




\begin{tabular}{l|l|l|l|}
\hline$-4050-4200 \mathrm{~m}$ & $1.76 \pm 1.79$ & $1.42 \pm 1.26$ & \\
$-4800 \mathrm{~m}$ & $1.76 \pm 2.07$ & $2.06 \pm 2.03$ & $1.21 \pm 1.23$ \\
$-5100-5200 \mathrm{~m}$ & $0.57 \pm 0.93$ & \multicolumn{3}{|l|}{} \\
\hline \multicolumn{4}{|l}{ All measured above relate to those measured on the first morning at each altitude except Borg } \\
Scores which were measured the on the evening before; P values refer to overall group \\
comparisons of marginal means for the apnoea versus control groups; $\mathrm{SpO}_{2}$ oxygen saturation, \\
\%; SL, sea level; RPE, rating of perceived exertion \\
\hline
\end{tabular}


Table 3 Results of Split-Level ANOVA on High altitude symptoms and Physiological measures

\begin{tabular}{|c|c|c|c|c|c|c|}
\hline & \multicolumn{2}{|c|}{ Effect of Apnoea } & \multicolumn{2}{|c|}{ Effect of Altitude } & \multicolumn{2}{|c|}{ Interaction } \\
\hline & $\mathbf{F}$ & $P$ value & $\mathbf{F}$ & $P$ value & $\mathbf{F}$ & P Value \\
\hline Heart rate & 0.39 & 0.54 & 5.84 & $<0.0001$ & 0.82 & 0.56 \\
\hline Systolic blood pressure & 2.65 & 0.12 & 4.59 & $<0.0001$ & 1.08 & 0.38 \\
\hline Diastolic blood pressure & 1.76 & 0.20 & 5.29 & $<0.0001$ & 0.41 & 0.18 \\
\hline $\mathrm{SpO}_{2}$ & 3.33 & 0.08 & 138.47 & $<0.0001$ & 1.34 & 0.24 \\
\hline Borg RPE Scores & 0.12 & 0.73 & 83.95 & $<0.0001$ & 0.93 & 0.48 \\
\hline Sleep scores & 1.07 & 0.31 & 0.73 & 0.62 & 0.69 & 0.66 \\
\hline GAD-7 & 0.90 & 0.35 & 5.46 & 0.006 & 1.45 & 0.24 \\
\hline Lake Louise Scores & 0.54 & 0.47 & 3.98 & 0.002 & 0.59 & 0.49 \\
\hline $\begin{array}{l}\text { All measured above relate } \\
\text { measured the on the eveni } \\
\text { Generalised Anxiety Diso }\end{array}$ & lose meas & $\begin{array}{l}\text { the first mo } \\
\text { n saturation }\end{array}$ & $\begin{array}{l}\text { ing at eac } \\
\% \text {; RPE, r }\end{array}$ & $\begin{array}{l}\text { itude except } \\
\text { of perceive }\end{array}$ & rg Scor & $\begin{array}{l}\text { hich were } \\
\text { D, }\end{array}$ \\
\hline
\end{tabular}




\section{Figures}

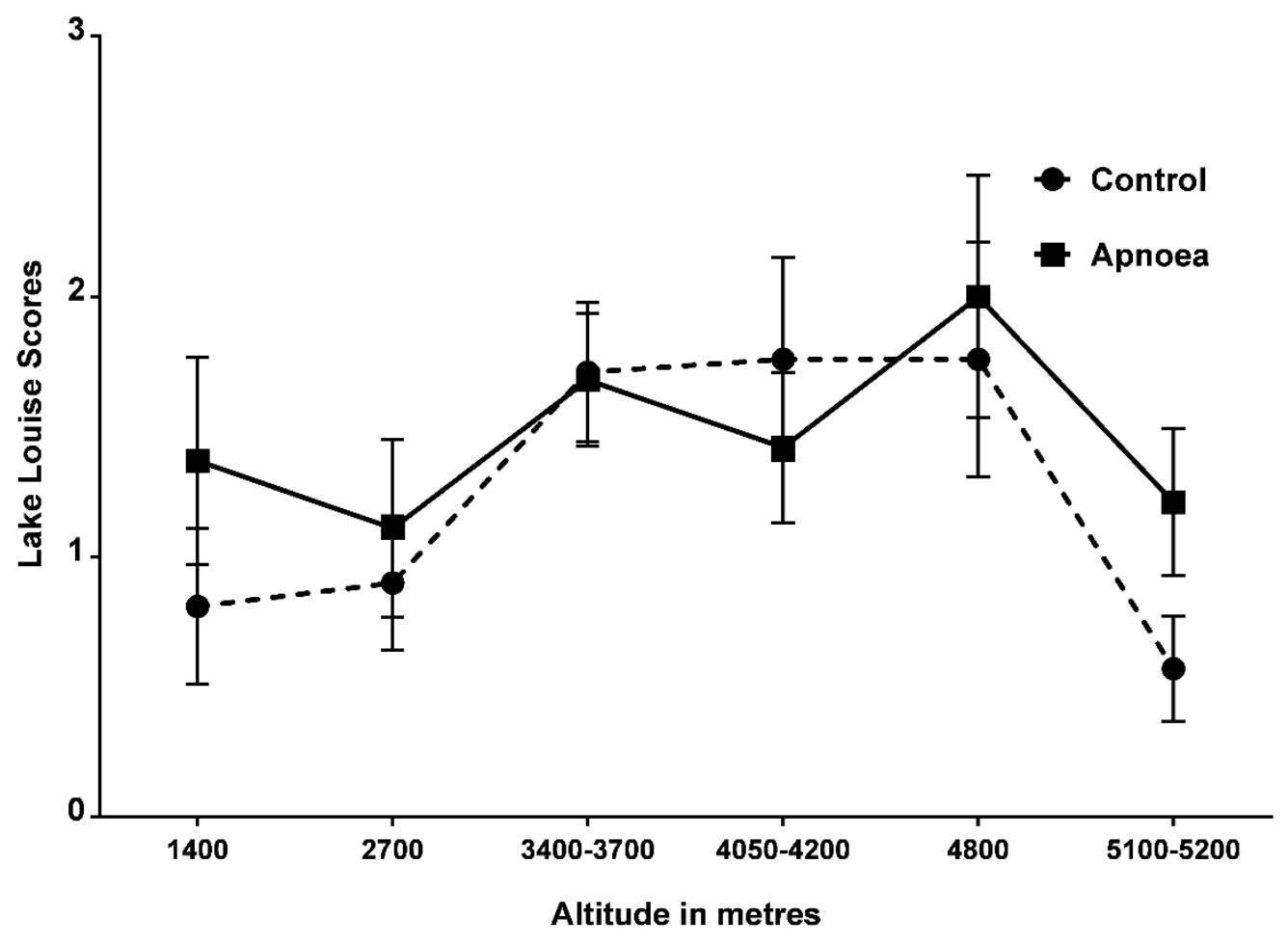

Figure 1 Comparative changes in Lake Louise Scores (mean \pm standard error of the mean) with increase high altitude among the control versus apnoea groups 


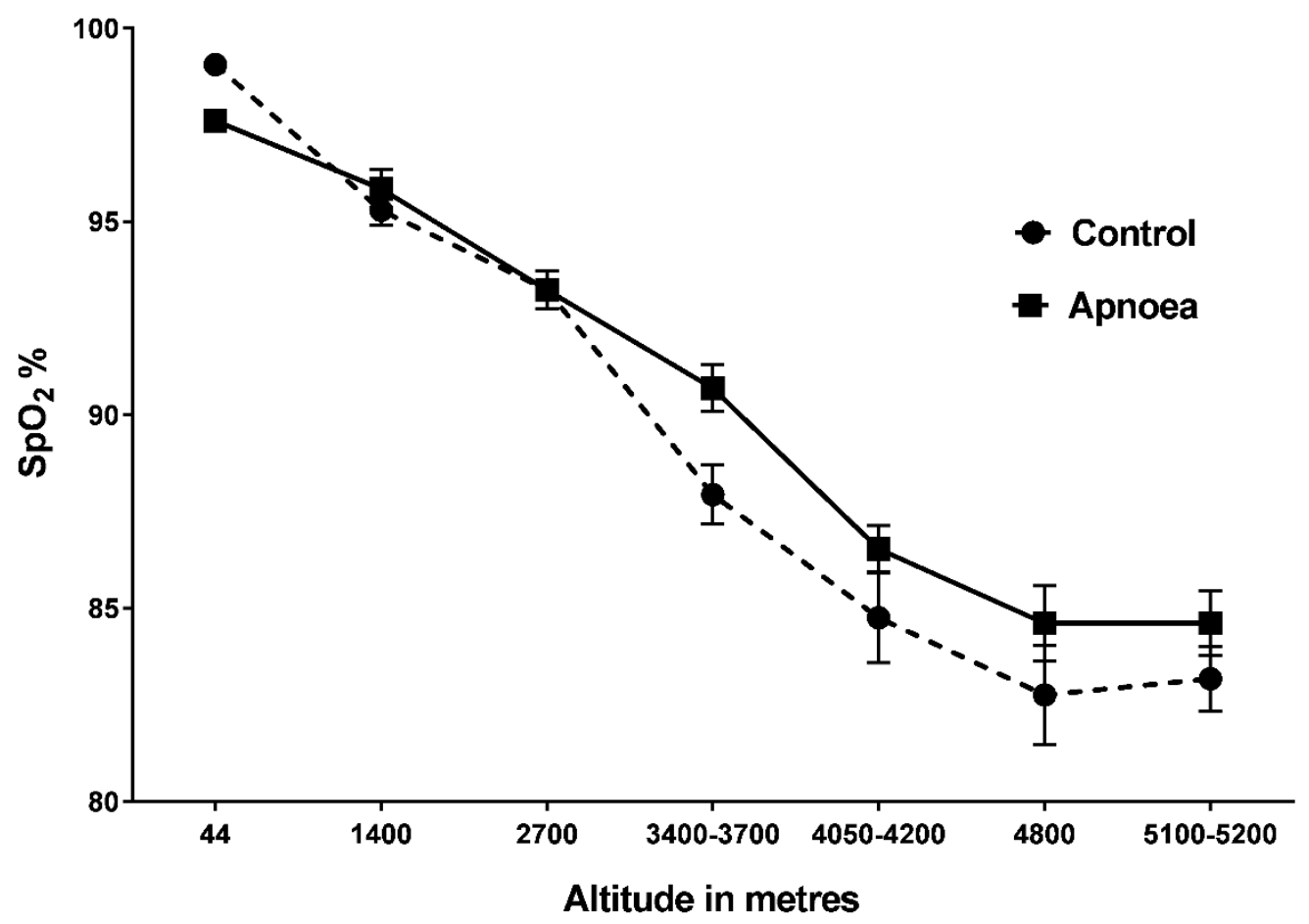

Figure 2 Comparative changes in $\mathrm{SpO}_{2}$ (mean \pm standard error of the mean) with increase high altitude among the control versus apnoea groups 